\title{
CARACTERÍSTICAS TOMOGRÁFICAS DA DISPLASIA FIBROSA CRANIOFACIAL: ESTUDO RETROSPECTIVO DE 14 CASOS*
}

\author{
Renato Assayag Botelho ${ }^{1}$, Olger de Souza Tornin ${ }^{1}$, Ilka Yamashiro ${ }^{2}$, Marcelo Carneiro \\ Menezes $^{1}$, Sérgio Furlan ${ }^{3}$, Maurício Ridelenski ${ }^{4}$, Rubens Yamashiro ${ }^{5}$, José Francisco Sales \\ Chagas $^{6}$, Ricardo Pires de Souza ${ }^{7}$
}

\begin{abstract}
Resumo OBJETIVO: Apresentar as várias formas de displasia fibrosa craniofacial em um estudo revisional com 14 exames de tomografia computadorizada. MATERIAIS E MÉTODOS: Foram selecionados 14 casos de displasia fibrosa craniofacial atendidos no Serviço de Cirurgia de Cabeça e Pescoço e Otorrinolaringologia e no Serviço de Diagnóstico por Imagem do Hospital Heliópolis, São Paulo, SP, entre 1991 e 2002. Foram avaliados, pelos autores, três dos achados tomográficos mais relevantes: o número de ossos acometidos, a apresentação radiológica predominante e a uni/bilateralidade da doença no crânio. RESULTADOS: Observou-se predomínio do padrão misto em relação à forma de apresentação da doença. Foram vistos, ainda, acometimento contíguo de dois ou mais ossos e equivalência entre casos de acometimento craniofacial unilateral e bilateral, possivelmente por conta dos pacientes serem, em sua maioria, portadores de displasia fibrosa do tipo monostótica. CONCLUSÃO: O conhecimento das características tomográficas da displasia fibrosa craniofacial é de grande importância para que seja feito planejamento cirúrgico adequado e seguimento pós-tratamento.

Unitermos: Displasia fibrosa óssea; Crânio; Face; Ossos faciais; Tomografia computadorizada por raios X.
\end{abstract}

Abstract Tomographic features of craniofacial fibrous dysplasia: a retrospective study of 14 cases.

OBJECTIVE: To describe the different presentations of craniofacial fibrous dysplasia in a revisional study of 14 computed tomography exams. MATERIALS AND METHODS: The authors have selected 14 cases of craniofacial fibrous dysplasia attended at the Head \& Neck Surgery/Otorhinolaryngology Service and Diagnostic Imaging Service of "Hospital Heliópolis", São Paulo, SP, Brazil, between 1991 and 2002. Three of the following most relevant tomographic findings were analyzed: number of bones affected, predominant radiological presentation and the disease uni- or bilaterality. RESULTS: A predominance of a mixed pattern was observed in relation to the disease presentation. Also, contiguous involvement of two or more bones was observed as well as equivalence between cases of unilateral and bilateral craniofacial involvement, possibly due the fact that most patients presented the monostotic type of fibrous dysplasia. CONCLUSION: The knowledge of tomographic features of craniofacial fibrous dysplasia is of paramount importance for surgical planning and post-treatment follow-up.

Keywords: Fibrous dysplasia of bone; Skull; Face; Facial bones; X-ray computed tomography.

\section{INTRODUÇÃO}

A displasia fibrosa é uma anomalia benigna do desenvolvimento, na qual a cavidade medular é substituída por material fibroso, osso entrelaçado e células em fuso $^{(1)}$. É observada, mais freqüentemente,

* Trabalho realizado no Serviço de Diagnóstico por Imagem do Hospital Heliópolis, São Paulo, SP.

1. Médicos Radiologistas, Mestrandos em Ciências da Saúde pelo Hospital Heliópolis.

2. Médica Radiologista, Mestre em Ciências da Saúde pelo Hospital Heliópolis.

3. Médico Radiologista

4. Médico Radiologista, Doutorando em Radiologia pela Universidade de São Paulo.

5. Médico Radiologista, Chefe do Setor de Tomografia Computadorizada do Hospital Heliópolis.

6. Médico Cirurgião, Professor do Curso de Pós-graduação do Hospital Heliópolis, Doutor em Medicina pela Universidade Federal de São Paulo-Escola Paulista de Medicina.

7. Coordenador da Residência Médica em Radiologia e Diagnóstico por Imagem do Hospital Heliópolis, Professor do Curso nas três primeiras décadas de vida ${ }^{(2,3)}$. Embora a displasia fibrosa seja uma doença de caráter benigno, há relatos de transformação maligna associada a essa forma de apresentação $^{(\mathbf{4 , 5})}$.

A região craniofacial é importante sítio de acometimento da doença, com destaque para a maxila e mandíbula ${ }^{(6-8)}$. O envolvimento dos ossos da face pode ocasionar espessamento e esclerose acentuados, freqüentemente com a obliteração de seios e órbitas, criando uma aparência "leonina" (leontíase óssea) ${ }^{(\mathbf{9})}$. Outras condições asso-

de Pós-graduação do Hospital Heliópolis, Doutor em Radiologia pela Universidade de São Paulo.

Endereço para correspondência: Dr. Renato Assayag Botelho. Rua Cônego Xavier, 276, $1^{\circ}$ andar, Secretaria do RX, Sacomã. São Paulo, SP, 04231-030. E-mail: assayag@uol.com.br

Recebido para publicação em 21/11/2005. Aceito, após revisão, em 30/11/2005. ciadas à displasia fibrosa nessa região são deformidades craniofaciais, má-oclusão dentária e diminuição da acuidade visual (esta última com indicação absoluta de tratamento cirúrgico).

Dentre os métodos de imagem, a tomografia computadorizada tem sido o mais usado para demonstrar a extensão e a radiodensidade que a displasia fibrosa assume nos ossos craniofaciais, sendo, portanto, de fundamental importância no planejamento cirúrgico e no seguimento longitudinal dos pacientes operados ${ }^{(\mathbf{1 0 , 1 1})}$. Os achados tomográficos da displasia fibrosa podem variar consideravelmente, dependendo da quantidade de tecido ósseo presente na lesão. Aparentemente, pode-se fazer uma divisão em três padrões de acometimento: o esclerótico, o cístico ou o misto ${ }^{(\mathbf{1 2})}$. 
No esqueleto, o padrão misto é o mais comumente encontrado ${ }^{(6,13)}$. Este padrão é caracterizado por áreas escleróticas associadas a áreas císticas ${ }^{(\mathbf{1 0})}$ (Figuras 1 e 2). O padrão cístico é caracterizado por áreas císticas ovaladas ou arredondadas, com bordas escleróticas $^{(6)}$ (Figuras 3 e 4). O padrão esclerótico é observado, freqüentemente, em pacientes mais jovens e consiste, radiograficamente, de opacidades em "vidro fosco" e expansão óssea (Figuras 5 e 6).

Este estudo busca demonstrar os aspectos tomográficos das principais formas de apresentação da displasia fibrosa através da análise de 14 exames de tomografia computadorizada de crânio e face de pacientes com diagnóstico firmado de displasia fibrosa craniofacial, comparando com os achados encontrados na literatura.

\section{MATERIAIS E MÉTODOS}

No período de 1991 a 2002, foram selecionados, retrospectivamente, 14 exames de tomografia computadorizada de crânio e face de pacientes com diagnóstico confirmado de displasia fibrosa craniofacial. Desses 14 pacientes, sete eram do sexo masculino $(50 \%)$ e sete eram do sexo feminino $(50 \%)$, com idade variando entre 8 e 33 anos. Os exames foram realizados em tomógrafos de terceira geração, com obtenção de cortes axiais e coronais. A espessura de corte e o incremento de mesa foram de $3 \mathrm{~mm}$ e $5 \mathrm{~mm}$, respectivamente.

Para a inclusão neste trabalho, os pacientes deveriam ser tratados no Serviço de Cirurgia de Cabeça e Pescoço do Hospital Heliópolis, ter confirmação anatomopatológica, não ter recebido tratamento prévio ou qualquer procedimento intervencionista antes da realização das imagens tomográficas. Durante a seleção, foram excluídos os exames realizados após intervenção cirúrgica e os de pacientes portadores de displasia fibrosa na forma de "querubismo".

Os casos foram avaliados por dois radiologistas, ambos membros titulares do Colégio Brasileiro de Radiologia e Diagnóstico por Imagem. Esses profissionais analisaram aspectos como número de ossos acometidos, apresentação radiológica predominante (a resposta deveria ser padrão esclerótico, cístico ou misto) e unilateralidade ou bilateralidade da doença no crânio, através da leitura dos exames.

\section{RESULTADOS}

Os resultados obtidos após a avaliação dos dois radiologistas estão evidenciados nas Tabelas 1, 2 e 3.

Tabela 1 Número de ossos acometidos pela displasia fibrosa.

\begin{tabular}{|c|c|c|}
\hline Ossos acometidos & Incidência & $(\%)$ \\
\hline Entre 1 e 3 ossos & $5 / 14$ & $(35,4 \%)$ \\
Entre 4 e 6 ossos & $4 / 14$ & $(28,6 \%)$ \\
Entre 7 e 9 ossos & $5 / 14$ & $(35,4 \%)$ \\
\hline
\end{tabular}

Tabela 2 Padrão tomográfico predominante.

\begin{tabular}{|l|c|c|}
\hline Padrão tomográfico & Incidência & (\%) \\
\hline Misto & $8 / 14$ & $(57,1 \%)$ \\
Cístico & $2 / 14$ & $(14,3 \%)$ \\
Esclerótico & $4 / 14$ & $(28,6 \%)$ \\
\hline
\end{tabular}

Tabela 3 Distribuição das lesões quanto à unilateralidade/bilateralidade.

\begin{tabular}{|l|c|c|}
\hline Lateralidade & Incidência & (\%) \\
\hline Lesões unilaterais & $7 / 14$ & $(50 \%)$ \\
Lesões bilaterais & $7 / 14$ & $(50 \%)$ \\
\hline
\end{tabular}

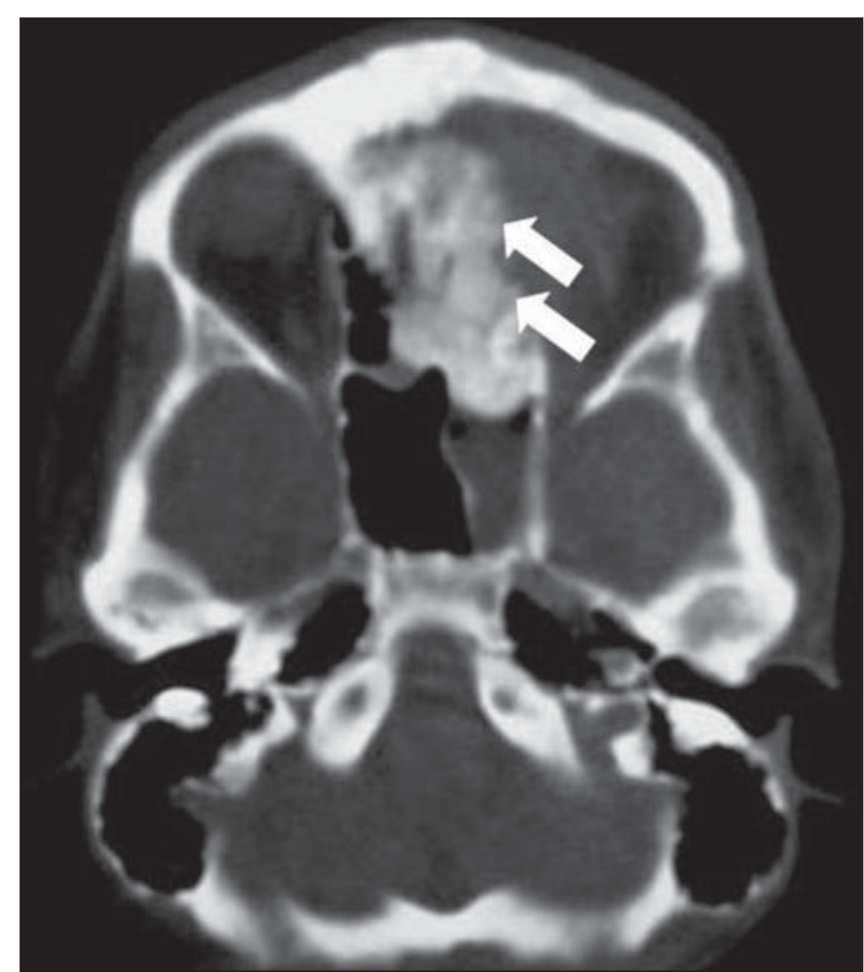

Figura 1. Tomografia computadorizada do crânio no plano axial, no nível do seio esfenoidal e células etmoidais. Evidencia-se displasia fibrosa no padrão misto na topografia de células etmoidais à esquerda (setas).

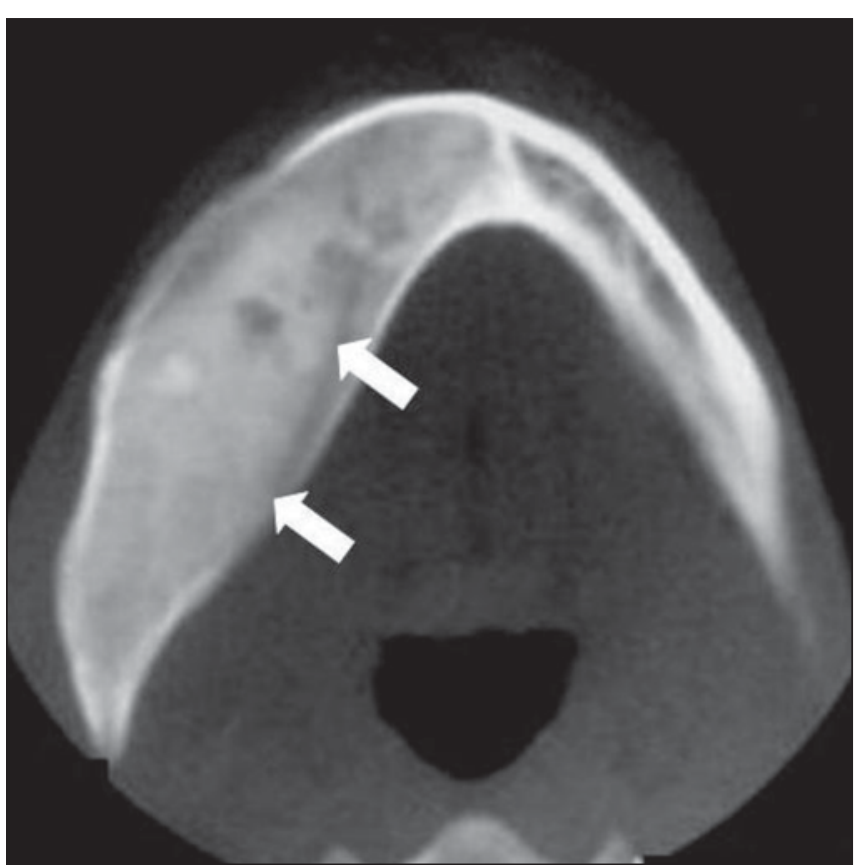

Figura 2. Tomografia computadorizada da mandíbula no plano axial. Demonstra-se displasia fibrosa no padrão misto no ramo mandibular à direita (setas). 


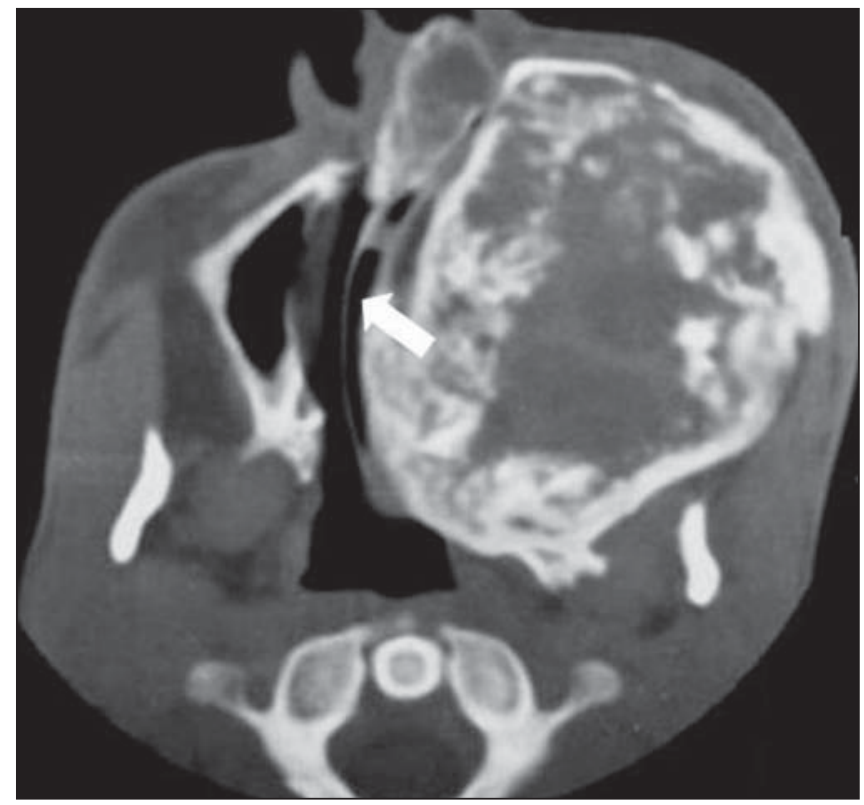

Figura 3. Tomografia computadorizada dos seios da face no plano axial. Percebe-se displasia fibrosa padrão cístico condicionando distorção da fossa nasal homolateral, com desvio do septo nasal para a direita (seta).

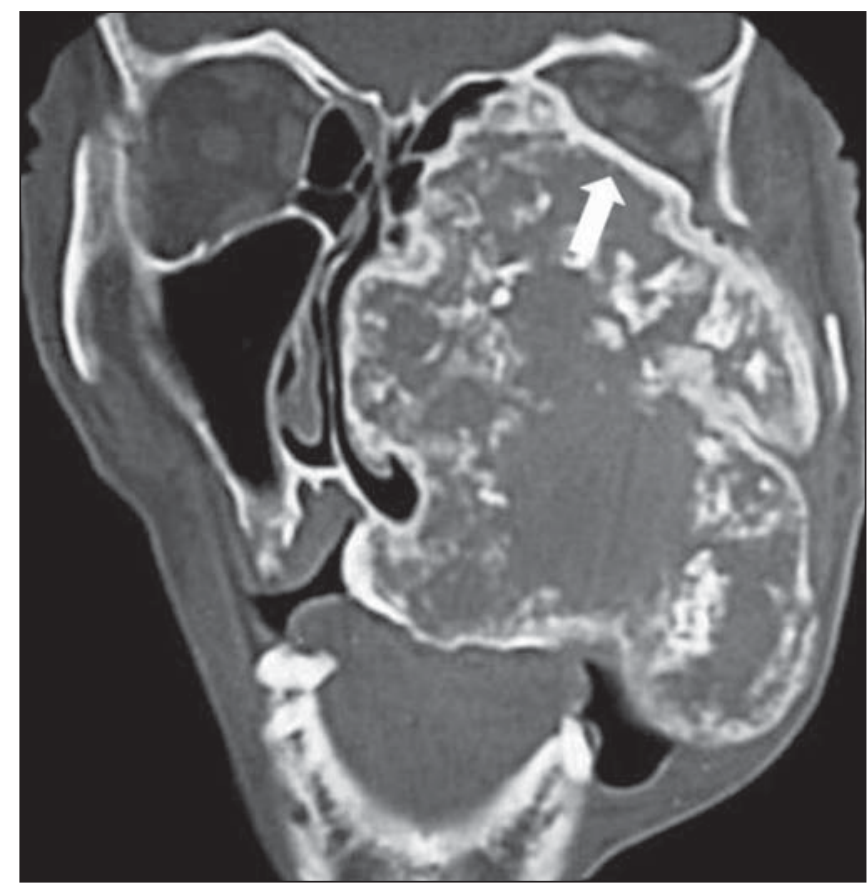

Figura 4. Tomografia computadorizada dos seios da face no plano coronal. Nota-se displasia fibrosa padrão cístico condicionando deformidade da parede óssea da cavidade orbitária esquerda (seta).

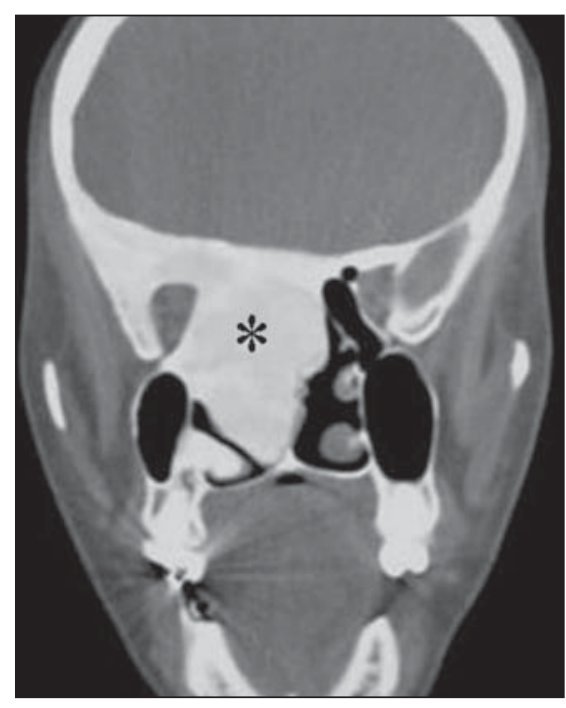

Figura 5. Tomografia computadorizada dos seios da face no plano coronal. Evidencia-se displasia fibrosa padrão esclerótico envolvendo células etmoidais, cavidade nasal e asa do osso esfenoidal à direita (asterisco).

\section{DISCUSSÃO}

A displasia fibrosa também pode ser dividida em dois grupos, quanto à forma de apresentação: monostótica e poliostótica.

A forma monostótica é aquela em que ocorre o acometimento de um osso ou de

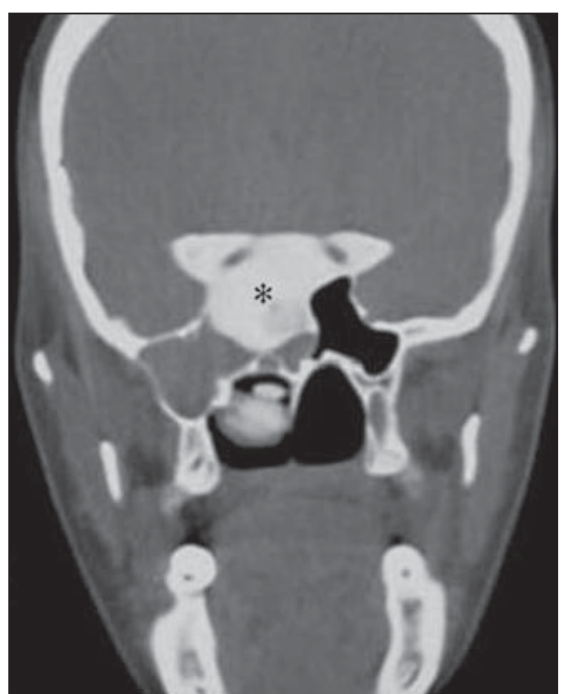

Figura 6. Tomografia computadorizada dos seios da face no plano coronal. Demonstra-se displasia fibrosa padrão esclerótico envolvendo a cavidade selar e clinóides (asterisco).

ossos contíguos ${ }^{(\mathbf{1 0})}$. É considerada a forma mais comum, correspondendo a aproximadamente $70 \%$ dos $\operatorname{casos}^{(\mathbf{1 0})}$. As faixas etárias acometidas são a de crianças e a de adultos jovens, sem predominância por sexo ${ }^{(6)}$. Na grande maioria das vezes ocorre a parada de crescimento das lesões com o fechamento da placa de crescimento $^{(\mathbf{1 4})}$. Clinicamente, a doença é caracterizada por aumento dos ossos envolvidos, com saliência das lâminas ósseas.

A forma poliostótica, por sua vez, é aquela que ocorre em dois ou mais ossos sem continuidade, e representa aproximadamente $30 \%$ de todos os casos. As faixas etárias acometidas são semelhantes às da forma monostótica, com predominância do sexo feminino. As manifestações clínicas são precoces e de início insidioso, podendo cursar com dor óssea. Quando esta forma de displasia fibrosa está associada com pigmentação cutânea "café-com-leite" e endocrinopatias, é conhecida como síndrome de McCune-Albright ${ }^{(14)}$. Quase todos os pacientes portadores de displasia fibrosa poliostótica apresentam envolvimento craniofacial, com predileção surpreendente por um lado do corpo ${ }^{(\mathbf{8 , 1 0})}$. Entretanto, a maioria dos pacientes que têm a doença nos ossos craniofaciais apresenta a forma monostótica ${ }^{(\mathbf{1 0})}$.

$\mathrm{Na}$ amostra estudada neste trabalho, a grande maioria de casos foi de pacientes que desenvolveram displasia fibrosa na forma monostótica. Por conta disso, os achados de comprometimento contíguo quando da presença da doença em dois 
ossos ou mais e a equivalência entre casos de acometimento craniofacial unilateral e bilateral são compatíveis com os resultados de estudos encontrados na literatura médica ${ }^{(\mathbf{1 0}, 12,15)}$. Embasados nesses trabalhos, encontramos também como padrão tomográfico predominante o misto.

\section{CONCLUSÃO}

O conhecimento dos diversos aspectos tomográficos da displasia fibrosa craniofacial é de grande importância para o radiologista geral, tendo em vista que os achados de imagem da doença são bastante característicos e que a tomografia computadorizada ocupa lugar de destaque no planejamento cirúrgico e no seguimento desta doença.

\section{REFERÊNCIAS}

1. Weissleder R, Rieumont MJ, Wittenberg J. Introdução ao diagnóstico por imagem. $2^{2}$ ed. Rio de Janeiro, RJ: Editora Revinter, 2004.

2. Jones SM, Kingston H, Mitra D, MacLeod TI, Bhalla AK. Coexisting polyostotic fibrous dysplasia and Paget's disease. Clin Exp Rheumatol 1996;14:187-190.

3. Levy ML, Chen TC, Weiss MH. Monostotic fibrous dysplasia of the clivus. A case report. J Neurosurg 1991;75:800-803.

4. Ruggieri P, Sim FH, Bond JR, Unni KK. Malignancies in fibrous dysplasia. Cancer 1994;73: 1411-1424.

5. Yabut SM Jr, Kenan S, Sissons HA, Lewis MM. Malignant transformation of fibrous dysplasia. A case report and review of the literature. Clin Orthop Relat Res 1988;(228):281-289.

6. Som PM, Curtin HD. Head and neck imaging. 4th ed. New York, NY: Mosby, 2004.

7. Valvassori GE, Maffe MF, Carter BL. Imaging of the head and neck. New York, NY: Thieme, 1995.

8. Greenspan A. Radiologia ortopédica. 3 a ed. Rio de Janeiro, RJ: Guanabara Koogan, 2001.
9. Eisenberg RL. Um atlas de diagnóstico diferen-

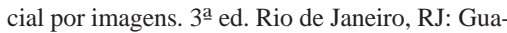
nabara Koogan, 1999.

10. Chen YR, Wong FH, Hsueh C, Lo LJ. Computed tomography characteristics of non-syndromic craniofacial fibrous dysplasia. Chang Gung Med J 2002;25:1-8.

11. MacDonald-Jankowski DS, Yeung R, Li TK, Lee KM. Computed tomography of fibrous dysplasia. Dentomaxillofac Radiol 2004;33:114-118.

12. Voegels RL, Thomé DC, Imamura R, Nakano EA, Butugan O, Miniti A. Displasia fibrosa da região da cabeça e pescoço: aspectos clínico e cirúrgico. Rev Bras Otorrinolaringol 1998;64(4 Pt 1):362365.

13. Resnick D. Diagnosis of bone and joint disorders. 4th ed. Philadelphia, PA: Saunders, 2002.

14. Contran RS, Kumar V, Robbins SL. Robbins Patologia estrutural e funcional. 5 $\underline{\underline{a}}$ ed. Rio de Janeiro, RJ: Guanabara Koogan, 1996.

15. MacDonald-Jankowski DS. Fibrous dysplasia in the jaws of a Hong-Kong population: radiographic presentation and systematic review. Dentomaxillofac Radiol 1999;28:195-202. 